\title{
The Hippocampus and Memory of Verbal and Pictorial Material
}

\author{
Andrew C. Papanicolaou, ${ }^{1,4}$ Panagiotis G. Simos, ${ }^{1}$ Eduardo M. Castillo, ${ }^{1}$ \\ Joshua I. Breier, ${ }^{1}$ Jeffrey S. Katz, ${ }^{2}$ and Anthony A. Wright ${ }^{3}$ \\ ${ }^{1}$ Vivian L. Smith Center for Neurologic Research, Department of Neurosurgery, University of Texas Health Science Center-Houston, Texas \\ 77030, USA; ${ }^{2}$ Psychology Department, Auburn University, Auburn, Alabama 36849, USA; ${ }^{3}$ Department of Neurobiology and Anatomy, University \\ of Texas Health Science Center, Houston, Texas 77030, USA
}

\begin{abstract}
Recognition of words and kaleidoscope pictures showed a double dissociation of left and right hippocampal activity using magnetic source imaging (MSI). MSI has advantages over alternative imaging techniques that measure hemodynamic changes for identifying regional changes in brain activity in real time and on an individual subject basis without the need for image subtraction. In this study, lists of words or kaleidoscope pictures were presented for memorization followed by tests of list items and foils during which brain activity was recorded. There was greater activation in the left than the right hippocampus with abstract nouns (e.g., relief) and greater activation in the right than the left hippocampus with kaleidoscope pictures. This dissociation was evident on a case by case basis. This study demonstrates the specialization of the two medial temporal lobe (MTL) regions, including the hippocampi, for mnemonic processing of verbal and pictorial items that are difficult to encode verbally.
\end{abstract}

The hippocampus has been a major focus of memory research since the seminal work of Scoville and Milner (1957). Human and animal lesion studies have shown that the hippocampus and other medial temporal lobe (MTL) structures are involved in at least some aspects of declarative memory (e.g., Milner et al. 1998). Despite considerable research, however, the specific role of the hippocampus in memory is not well understood (e.g., Stark and Squire 2000a). Functional neuroimaging is beginning to contribute evidence about the role of MTL structures in memory. For example, several studies using functional magnetic resonance imaging (fMRI) showed increased MTL activity during encoding of verbal or nonverbal material, and the degree of activity was directly related to memory performance (Gabrieli et al. 1997; Martin et al. 1997; Wagner et al. 1997; Brewer et al. 1998; Fernandez et al. 1998; Kelley et al. 1998).

Fewer neuroimaging studies have investigated retrieval processes. Perhaps early failures contributed to the delay of investigating this important issue (Grasby et al. 1993; Kapur et al. 1994; Shallice et al. 1994; Buckner et al. 1995; Fletcher et al. 1995). Recently, however, some studies have shown hippocampal activity during retrieval. Two studies found activity in across-modality conditions in which the study items were pictures and the test items were words that named objects in the pictures (Gabrieli et al. 1997; Stark and

${ }^{4}$ Corresponding author

E-MAIL Andrew.C.Papanicolaou@uth.tmc.edu; FAX 713-7977590.

Article and publication are at http://www.learnmem.org/cgi/doi/ $10.1101 / \mathrm{lm} .44302$.
Squire 2000b). Another study found activity in same-modality conditions in which test and study items were either both pictures or both words (Stark and Squire 2000a). This last result is important for its theoretical implications about the hippocampus and memory in general. If retrieval involves many of the same circuits used in encoding information, then the hippocampus would have to be actively involved in retrieval just as it is in encoding. The reason why such hippocampal activity has only recently been shown for memory retrieval is unclear and remains speculative. A better understanding of the conditions that reveal hippocampal activity during memory retrieval may lead to a better understanding of the role of the hippocampus in memory.

Also important for a better understanding of the role of MTL and of the hippocampus in memory would be the ability of imaging the activation of these structures in individual subjects while they process individual stimuli. Of all currently available functional imaging modalities, only magnetic source imaging (MSI) readily offers this capability. MSI, also referred to as magnetoencephalography (MEG), is based on the recording of changes in magnetic flux at the scalp that are virtually contemporaneous with changes in neuronal activity that occur in real time during any information-processing task. As such, magnetic-flux changes do not lag several seconds or minutes behind the task-relevant neuronal activity, as do blood-flow changes measured by fMRI and positron emission tomography (PET). Moreover, MSI does not require subtraction of images between baseline and experimental conditions that may involve questionable assumptions. Like electroencephalography (EEG), MSI

LEARNING \& MEMORY 9:99-104 @ 2002 by Cold Spring Harbor Laboratory Press ISSN1072-0502/02 \$5.00

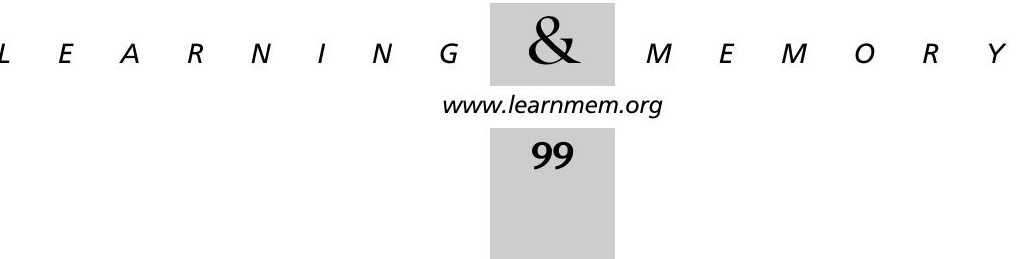


recordings have essentially instantaneous temporal resolution. Unlike EEG, however, MSI recordings are not influenced by volume conduction and therefore allow relatively precise location of intracranial sources (Papanicolaou et al. 1991; Breier et al. 1998; Simos et al. 1998; Breier et al. 1999b; Papanicolaou et al. 1999; Simos et al. 1999).

In the present study we recorded changes in magnetic flux during performance of two episodic memory tasks. In one task, participants were instructed to study printed abstract nouns (e.g., cost, menace, mood, and relief). Such verbal material has been shown to be processed primarily in the left hemisphere (e.g., Milner 1971). The second task involved pictorial material that is not amenable to verbal processing (Wright et al. 1990). Such nonverbal material has been shown to be processed primarily in the right hemisphere (e.g., Milner 1971). Brain activation maps were constructed during a subsequent recognition phase, which required the incidental retrieval of information memorized during the learning phase, regarding each stimulus. From previous evidence showing hemispheric specialization of verbal and nonverbal material, we hypothesized that there should be primarily left hippocampal activity during the recognition phase involving abstract nouns and primarily right hippocampal activity during the recognition phase involving kaleidoscope pictures. We were encouraged in this prediction by a recent fMRI study showing that encoding of verbal material produced activity that was lateralized primarily to the left MTL, and encoding of unnamable pictures (unfamiliar faces) produced activity that was lateralized primarily to the right MTL (Kelley et al. 1998).

\section{RESULTS}

The composite map of activity sources for the 12 participants and all activity sources that occurred in all the $0.4-\mathrm{cm}$ brain scan slices that traverse either the left or the right MTL region in each participant are shown in Figure 1. Activity sources for kaleidoscope pictures are shown in yellow, and activity sources for abstract words are shown in red. The results show that the majority of activity sources for words occurred in the left MTL, and that the majority of activity for kaleidoscope pictures occurred in the right MTL. On average, activity in the right MTL during the picture task was 2.28 times greater than activity in the left MTL. More than $85 \%$ of this activity occurred within, or directly adjacent to,
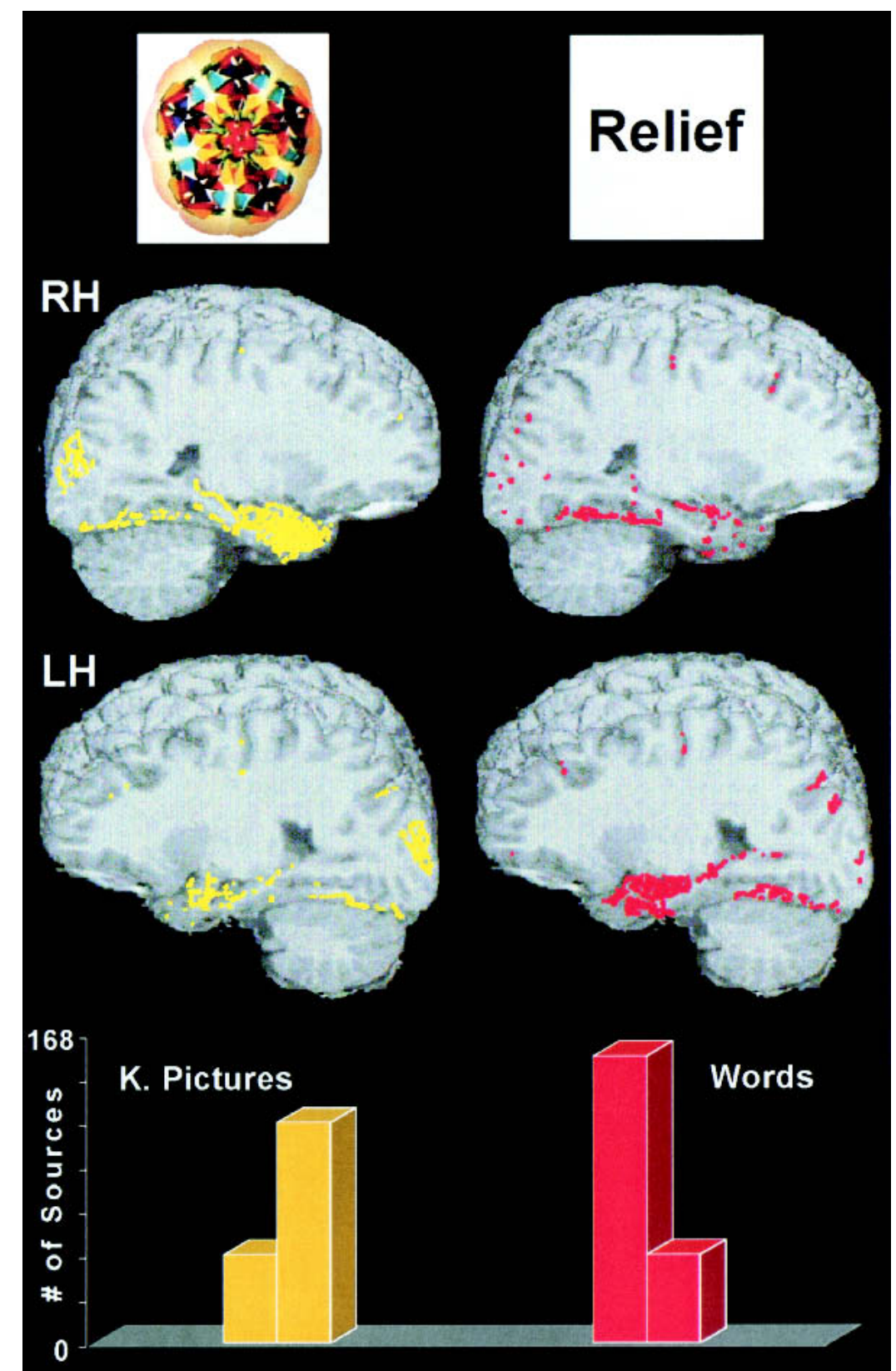

\section{MTL ACTIVITY SOURCES}

Figure 1 Top: Examples of the kaleidoscope pictures (left) and words (right) used in memory testing. Middle: Brain scan composite images for 12 subjects showing sources of activity separately for the right $(\mathrm{RH})$ and the left hemisphere $(\mathrm{LH})$ and for kaleidoscope pictures (yellow) and words (red). Bottom: Bar graphs showing the total number of activity sources in the medial temporal lobes (MTL) of the RH and LH for kaleidoscope (K) pictures (yellow) and words (red) for the group of 12 subjects.

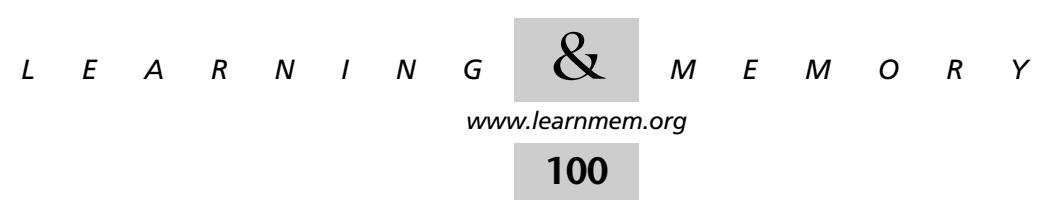


the hippocampus proper. ${ }^{1}$ This is the dense yellow cluster of activity shown in the lower-central region of the upper left-hand panel. Conversely, left MTL activity during the word task was 3.1 times greater than right-hemisphere MTL activity. Twelve percent of the total activity in the right hemisphere during picture processing occurred in the right MTL compared with only $6 \%$ of the total activity in the left hemisphere. The reverse pattern was found in the word task, with $14 \%$ of the total number of activity sources in the left hemisphere localized in MTL structures and only $9.6 \%$ in the right hemisphere. More than $85 \%$ of this activity occurred within, or directly adjacent to, the hippocampus. This is the dense red cluster of activity shown in the lowercentral region of the lower right-hand brain map.

Individual results for the 12 participants are shown in Table 1 . These individual results show essentially the same double dissociation of hippocampal activity shown by the group data and thus demonstrate the consistency of the findings. All subjects showed greater left than right MTL and hippocampal activity during the verbal condition. Nine of the 12 participants showed greater right than left MTL and hippocampal activity during the nonverbal condition. All subjects showed changes toward more right than left MTL activity with kaleidoscope pictures compared with words. Statistical analyses confirmed the double dissociation for words and kaleidoscope pictures. A two-way repeated measures analysis of variance (ANOVA) of the results in Table 1 showed an interaction between stimulus type and hemisphere, $F(1,11)=43.8, P<.00004$. Other ANOVAs supported the differences shown in the bar graphs of Figure 1; there was more right than left MTL activity during the retrieval test with kaleidoscope pictures, $F(1,11)=12.1$, $P<.005$, and more left than right MTL activity during the retrieval test for words, $F(1,11)=21.7, P<.0007$. In these analyses, the data for targets and foils and for both incorrect and correct identifications were collapsed. Separate analyses for targets versus foils and for correct versus incorrect identifications did not reveal any systematic differences. To further explore the relation between MSI data and individual performance, we computed a series of correlation coefficients between the number of activity sources in the left or right MTL and the percentage of correctly identified target stimuli in each task. None of these tests reached statistical significance, ranging between .26 (correlation between degree of activity in the right MTL and percentage of correctly identified kaleidoscope targets) and .35 (correlation between degree of activity in the left MTL and percentage of correctly identified word targets). The mean percentage of correctly identified target stimuli was $95 \%$ for words and $90 \%$ for kaleidoscope pictures, $P>.05$.

${ }^{1}$ Locating activity within or adjacent to the hippocampus approaches the spatial resolution limits $(\sim \pm 0.5 \mathrm{~cm})$ of MSI (Papanicolaou et al. 1999; Simos et al. 1999), as well as fMRI (Stark and Squire 2000a,b)
Table 1. Activity Sources in the Medial Temporal Lobe for Individual Subjects during Retrieval of Kaleidoscope Pictures and Words

\begin{tabular}{lccrrr}
\hline & \multicolumn{2}{c}{ Kaleidoscope pictures } & & \multicolumn{2}{c}{ Words } \\
\cline { 2 - 3 } \cline { 5 - 6 } Subjects & Left MTL & Right MTL & & Left MTL & Right MTL \\
\hline 1 & 6 & 15 & & 4 & 3 \\
2 & 2 & 6 & & 2 \\
3 & 2 & 6 & & 13 & 0 \\
4 & 15 & 26 & & 9 & 0 \\
5 & 4 & 13 & & 16 & 7 \\
6 & 0 & 14 & & 30 & 17 \\
7 & 2 & 9 & & 2 & 0 \\
8 & 6 & 3 & & 32 & 7 \\
9 & 0 & 10 & & 12 & 2 \\
10 & 6 & 5 & & 2 & 0 \\
11 & 2 & 3 & & 13 & 0 \\
12 & 5 & 5 & & 10 & 4 \\
\hline
\end{tabular}

MTL, medial temporal lobes.

The relative timing of activation across three brain regions where activity was reliably detected in every participant (MTL, temporoparietal, and visual occipital) and task is outlined in Figure 2. Individual brain activation profiles obtained during the word memory task resembled those observed in previous MSI studies in the context of silent reading tasks (Breier et al. 1998). They feature initial activation of the mesial occipital cortices bilaterally (within the first 150 msec after stimulus onset), followed by activity in basal temporal cortices predominantly in the left hemisphere (starting within $200 \mathrm{msec}$ poststimulus onset). In the next several hundred milliseconds, the profiles entailed activation of posterior temporal and inferior parietal areas as well as mesial temporal lobe regions, predominantly in the left hemisphere. The activation profiles in response to kaleidoscope stimuli were similar during the first $150 \mathrm{msec}$ after stimulus onset in that they featured bilateral occipital activity. Activation of basal temporal regions was then observed, followed by activity in posterior temporal and inferior parietal regions, predominantly in the right hemisphere. On average, this activity peaked earlier than activity in homotopic left hemisphere areas during the word task (250 vs. $400 \mathrm{msec}$ poststimulus onset). This difference, however, did not reflect earlier onset latencies in temporoparietal areas in the kaleidoscope (210 msec) compared with the word task (300 msec), $\mathrm{t}(11)=.15, P>.5$, and is probably associated with the fact that processing of the kaleidoscope stimuli entailed reactivation of occipital visual areas, which was not observed in the word task. This late activity in occipital areas peaked, on average, at $\sim 400 \mathrm{msec}$ after stimulus onset and coincided temporally with the initial "wave" of activity in the right MTL. Right MTL activation started to become apparent at $300 \mathrm{msec}$ and was sustained for the next $450 \mathrm{msec}$. In comparison, activity in the left

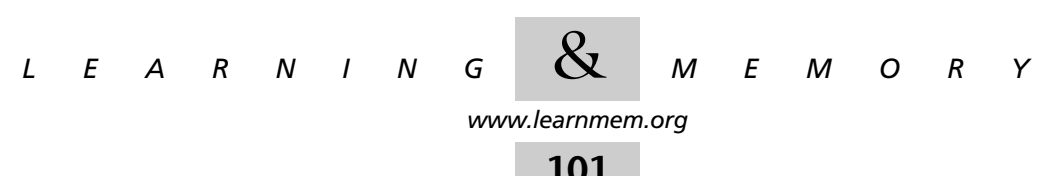



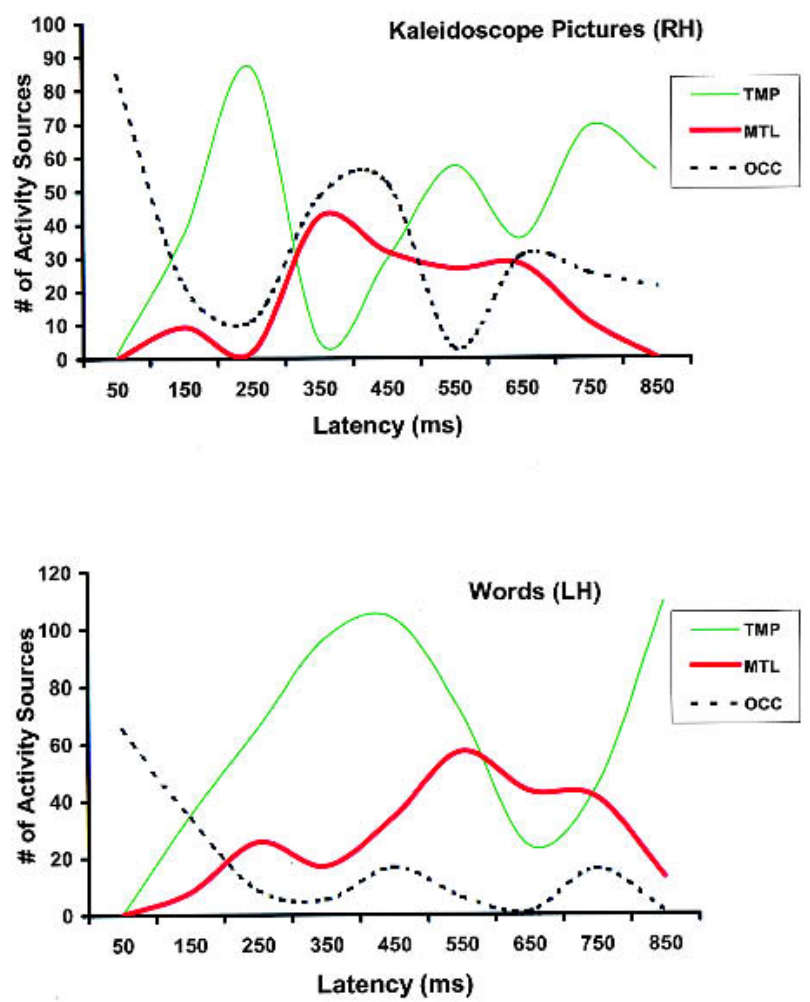

Figure 2. The temporal course of activation in three brain regions, temporoparietal (TMP), medial temporal (MTL), and occipital (OCC), during the picture task in the right hemisphere $(\mathrm{RH}$, top panel) and in the corresponding regions in the left hemisphere (LH) for the verbal task (lower panel).

MTL during word processing first became noticeable at 250 msec and was sustained until $\sim 800 \mathrm{msec}$. Task differences in the mean onset (300 vs. $250 \mathrm{msec}$ ) or offset of MTL activation (750 vs. $800 \mathrm{msec})$ were not statistically significant $(P>.1)$.

\section{DISCUSSION}

The primary finding of this study was a double dissociation in the degree of neurophysiological activity in the MTL as a function of stimulus type (abstract words vs. kaleidoscope pictures). This effect was obtained during the recognition phase of the same episodic memory task. Although this task did not involve active recall, ample activity in the hippocampus proper was observed that accounted for $9.6 \%$ to $14 \%$ of the total activity in the "dominant" hemisphere for a particular task. Another potentially interesting finding in the present study was that the degree of MTL activity did not vary consistently as a function of task performance and was found in response to stimuli that were correctly identified as targets as well as to stimuli that were not identified. Taken together, these findings are consistent with the idea that the observed material-specific activity in the MTL may serve as an index of the engagement of neural processes that are involved in recognition, rather than reflecting the outcome of this function. Such processes include visual analysis and the mapping of salient stimulus features on stored representations, that is, incidental retrieval. The latter process is necessary to ascertain whether a particular stimulus has been perceived previously during the task. As MSI allows virtually instantaneous localization of activity sources, neurophysiological activity associated primarily with the initial processing of the stimuli can be distinguished from later activity that is more likely to reflect processes involved in memory function. Given that all MTL activity sources observed in this study occurred after the initial activation of primary and modality-specific association cortices (i.e., after $200 \mathrm{msec}$ poststimulus onset), it is likely to reflect, at least in part, neural operations involved in memory retrieval.

The time lag between neuronal activity and hemodynamic changes (e.g., PET and fMRI) would not allow the activity of these different processes to be separated, which is why attempts are often made to study these processes separately in blocks of trials (e.g., Kelley et al. 1998; Stark and Squire 2000a,b). MSI also has the advantage that active sources are directly located and thereby avoids the issue of which baseline activity should be subtracted from activity in the experimental condition and the issue of explaining negative activity that might result from this subtraction. Imaging techniques that depend on blood-flow changes (e.g., PET and fMRI) are, however, well suited to the study of encoding performed in a repetitive block (e.g., a study list).

Notably, a similar hemispheric dissociation in the degree of late MTL activity has been observed in a previous MSI study in the context of a visual pseudoword-rhyming and a pattern-matching task (Breier et al. 1999a). In that study, lateralized activity in MTL was observed during a short time retention interval $(1.5 \mathrm{sec})$. In agreement with these results, a recent fMRI study found that encoding of verbal material resulted in hemodynamic changes primarily in the left MTL, whereas encoding of unnamable pictures (unfamiliar faces) was associated primarily with activity changes in the right MTL (Kelley et. al. 1998). Taken together, these studies show that hippocampal activity is not passive in nature but is directly related to the particular stimulus material in both retrieval and encoding.

\section{METHODS}

\section{Subjects}

Twelve adult volunteers, nine male and three female, who were students or employees at the University of Texas Medical SchoolHouston, participated in the study. None of the participants had a history of neurologic or psychiatric disorder, learning disability, or visual impairment. In addition, all participants were right-handed with English as their primary language. They were paid \$30 for their participation. The University of Texas Institutional Review Board had approved this study and all subjects were asked to sign a consent form after the nature of the procedures involved had been explained to them.

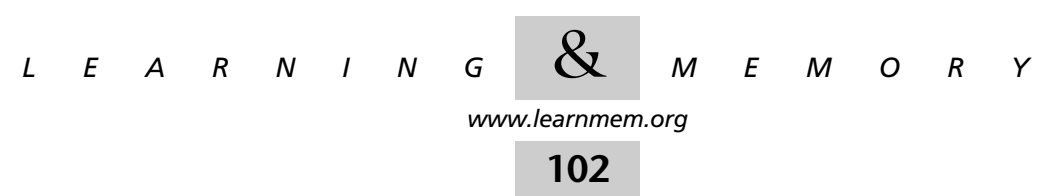




\section{Procedures}

For the learning phase, each participant was presented with a list of either 33 words or 33 kaleidoscope pictures for study. During the list presentation, no recordings were made. Following the list presentation, MSI recordings were conducted during retrieval of the items in a recognition memory format. This test phase consisted of three blocks with 43 trials in each block. In each block, the 33 list items were randomly mixed with 10 foils. Foils were different but qualitatively similar to list items. In previous studies we have found that this relative proportion of targets/foils produces the most stable (i.e., associated with a higher degree of test-retest reliability and intersubject consistency) activation profiles (Breier et al. 2000). In addition, this protocol produces activation profiles that are in closest agreement with the results of invasive functional brain-mapping procedures, including intracarotid sodium amytal (Breier et al. 1999b, 2001) and electrocortical stimulation (Simos et al. 1999).

The 10 foils were different in each block. Stimuli were presented for 1 sec with a 3 or 4 sec (random) delay between items. During the entire recording session that lasted $20 \mathrm{~min}$, participants were instructed to remain perfectly motionless except when raising an index finger to indicate that an item was from the study list. Use of the right or left index finger was counterbalanced across participants. The order of testing with words and kaleidoscope pictures was also counterbalanced across participants in two separate daily sessions.

MSI data were recorded in a magnetically shielded chamber (Vacuumschmelze $\mathrm{GmbH}$ ) with a whole-head neuromagnetometer (Magnes WH2500, 4-D Neuroimaging, Inc.) that consisted of 148 axial magnetometer coils placed in a cryogenic container. Stimuli were projected onto the chamber ceiling at a distance of $1.5 \mathrm{~m}$ from the participants and subtended a visual angle of $1^{\circ}$ to $3^{\circ}$ horizontally and $0.5^{\circ}$ vertically. Standard analysis techniques resulted in images displaying the location of MSI-derived equivalent point-dipole sources that represent brain activity during task performance (e.g., Sarvas 1987; Papanicolaou et al. 1991; 1999; Nakasato et al. 1995; Breier et al. 1998; 1999a; Simos et al. 1998; 1999). To identify the intracranial sources of the recorded event-related magnetic fields, a mathematical model was used that considered the intracranial activity sources (sets of active neurons) as equivalent to physical current dipoles (Sarvas 1987) and provided an estimate of the location and strength of these sources at successive 4 -msec intervals during the temporal evolution of a given magnetic response. Activity sources were considered acceptable if they were associated with a correlation coefficient of 0.9 or greater between observed and predicted magnetic flux distributions. There is currently considerable data supporting the validity of the single-moving dipole model for reliably localizing and lateralizing neurophysiological activity associated with language function. At our center, MSI-derived activation maps correspond very closely with the results of invasive functional brain-mapping techniques in patients with brain insult who were candidates for neurosurgical intervention, including the intracarotid sodium amytal procedure and electrocortical stimulation mapping (Breier et al. 1999b, 2001; Papanicolaou et al. 1999; Simos et al. 1999).

The event-related magnetic field waveform has an early component ( $<150 \mathrm{msec}$ poststimulus) that is believed to represent primary sensory processing and late components $(150-800 \mathrm{msec}$ poststimulus onset) that represent cognitive processing in higher-order association cortices (Papanicolaou et al., 1991; 1999; Breier et al. 1998; 1999b; Simos et al. 1998; 1999). In this study we focused on late components because we were not primarily interested in the initial reaction of the primary sensory cortex to the stimuli but in the brain mechanisms involved in the mapping and retrieval of task-related stored information regarding the stimuli. The number of reliably localized activity sources in MTL in each hemisphere served as the dependent measure in the statistical analyses. Activity sources in temporoparietal (including the posterior portion of the superior and middle temporal gyri and the supramarginal gyrus) and occipital areas (including visual areas 17 and 18) were also taken into account. This measure is the most reliable and valid index of the degree of regional cerebral activation, specific to various language operations in several studies involving neurologically intact volunteers and patients (Breier et al. 1999b; 2001; Papanicolaou et al. 1999; Maestú et al. 2001). Late activity sources were also found in temporoparietal areas (posterior portion of the superior and middle temporal gyri and the supramarginal gyrus) and in the occipital extrastriate areas.

For six of the participants, their brain activity sources were projected onto their own structural MRI scans. The location estimates of each "dipolar" activity source were specified with reference to a Cartesian coordinate system, anchored on three fiducial points on the head (the nasion and the external meatus of each ear). The anatomical location of activity sources was determined after coregistering MSI coordinates onto the subject's structural MRI scan. This was achieved by marking the same MSI fiducial points with vitamin pills during the MRI scans. In this way, activity sources that account for magnetic flux measurements indicate the location of brain areas activated at each consecutive 4-msec time frame in response to the stimuli. For the other six participants, their activity sources were projected onto MRIs that were matched to each subject according to three criteria: (1) the head size (circumference) had to be within $0.5 \mathrm{~cm}$, (2) the distance between the nasion and the origin of the Cartesian coordinate system had to be within $0.5 \mathrm{~cm}$ and (3) the overall shape of the scalp had to be within $0.5 \mathrm{~cm}$ at all points. A standard MRI atlas of the human brain (Damasio 1995) was then used as a reference for the identification of the cerebral structures where sources were localized. In addition to examining individual brain activation profiles, a composite map representing MTL activity across all 12 participants was derived by dividing the outline of the mesial temporal lobe in six consecutive segments (on each sagittal slice) for each scan, computing the number of activity sources in each segment, and then overlaying the sum for each segment onto a "model" MRI. This method preserves any gradients in the distribution of activity sources in the rostrocaudal axis.

\section{ACKNOWLEDGMENTS}

This research was supported by grants NSF REC-9979968, NIH NS37941-01, and NIH DA10715 to Dr. Papanicolaou. The authors thank Craig Stark for his thoughtful and encouraging comments on an earlier version of this manuscript.

The publication costs of this article were defrayed in part by payment of page charges. This article must therefore be hereby marked "advertisement" in accordance with 18 USC section 1734 solely to indicate this fact.

\section{REFERENCES}

Breier, J.I., Simos, P.G., Zouridakis, G., and Papanicolaou, A.C. 1998. Relative timing of neuronal activity in distinct temporal lobe areas during a recognition memory task for words. J. Clin. Exper. Neuropsychol. 20: 782-790. 
1999a. Temporal course of regional activation associated with phonological decoding. J. Clin. Exper. Neuropsychol. 21: 465-476.

Breier, J.I., Simos, P.G., Zouridakis, G., Wheless, J.W., Willmore, L.J., Constantinou, J.E., Maggio, W.W., and Papanicolaou, A.C. 1999b. Language dominance determined by magnetic source imaging: A comparison with the Wada Procedure. Neurology 53: 938-945.

Breier, J.I., Simos, P.G., Zouridakis, G., and Papanicolaou, A.C. 2000. Lateralization of activity associated with language function using magnetoencephalography: A reliability study. J. Clin. Neurophys., 17: 503-510.

Breier, J.I., Simos, P.G., Wheless, J.W., Constantinou, J.E.C., and Papanicolaou, A.C. 2001. Hemispheric language dominance in children determined by magnetic source imaging. J. Child Neurol. 16: 124-130.

Brewer, J.B., Zhao, Z., Glover, G.H., and Gabrieli, J.D.E. 1998. Making memories: Brain activity that predicts whether visual experiences will be remembered or forgotten. Science 281: 1185-1187.

Buckner, R.L., Petersen, S.E., Ojemann, J.G., Miezin, F.M., Squire, L.R., and Raichle, M.E. 1995. Functional anatomical studies of explicit and implicit memory retrieval tasks. J. Neurosci. 15: 12-29.

Damasio, H. 1995. Human brain anatomy in computerized images. Oxford University Press, NY.

Fernandez, B., Weyerts, H., Schrader-Bolsche, M., Tendolkar, I. Smid, H.G., Templemann, C., Hinrichs, H., Scheich, H., Elger, C.E., Mangun, G.R., et al. 1998. Successful verbal encoding into episodic memory engages the posterior hippocampus: A parametrically analyzed functional magnetic resonance imaging study. J. Neurosci. 18: 1841-1847.

Fletcher, P.C., Frith, C.D., Grasby, P.M., Shallice, T., Frackowiak, R.S.J., and Dolan, R.J. 1995. Brain systems for encoding and retrieving auditory-verbal memory: An in vivo study in humans. Brain 118: $408-416$

Gabrieli, J.D.E., Brewer, J.B., Desmond, J.E., and Glover, G.H. 1997. Separate neural bases of two fundamental memory processes in the human medial temporal lobe. Science 276: 264-266.

Grasby, P.M., Frith, C.D., Friston, K.J., Bench, C., Frachowiak, R.S., and Dolan, R.J. 1993. Functional mapping of brain areas implicated in auditory-verbal memory function. Brain 116: 1-20.

Kapur, S., Craik, F.I.M., Tulving, E., Wilson, A.A., Houle, S., and Brown, G.M. 1994. Neuroanatomical correlates of encoding in episodic memory: Levels of processing effect. Proc. Natl. Acad. Sci. 91: 2008-2011.

Kelley, W.M., Miezin, F.M., McDermott, K.B., Buckner, R.L., Raichle, M.E., Cohen, N.J., Ollinger, J.M., Akbudak, E., Conturo, T.E., Snyder, A.Z., et al. 1998. Hemispheric specialization in human dorsal frontal cortex and medial temporal lobe for verbal and nonverbal memory encoding. Neuron 20: 927-936.

Maestú, F., Fernández, A., Simos, P.G., Gil-Gregorio, P., Amo, C., Rodriguez, R., Arrazola, J., and Ortiz, T. 2001. Spatio-temporal patterns of brain magnetic activity during a memory task in Alzheimer's disease. Neuroreport 12: 3917-3922.
Martin, A., Wiggs, C.L., and Weisberg, J.W. 1997. Modulation of human medial temporal lobe activity by form, meaning, and experience. Hippocampus 7: 587-593.

Milner, B. 1971. Interhemispheric differences in the localization of psychological processes in man. Br. Med. Bull. 27: 272-277.

Milner, B, Squire, L.R., and Kandel, E.R. 1998. Cognitive neuroscience and the study of memory. Neuron 20: 445-468.

Nakasato, N., Fujita, S., Seki, K., Kawamura, T., Matani, A., Tamura, I., Fujiwara, S., and Takashi, Y. 1995. Functional localization of bilateral auditory cortices using an MRI-linked whole head magnetoencephalography (MEG) system. Electroencephal. Clin. Neurophysiol. 94: 183-190.

Papanicolaou, A.C., Rogers, R.L., and Baumann, S.B. 1991. Applications of magnetoencephalography to the study of cognition. Ann. N.Y. Acad. Sci. 620: 118-127.

Papanicolaou, A.C., Simos, P.G., Breier, J.I., Zouridakis, G., Willmore, L.J., Wheless, J.W., Constantinou, J.E., Maggio, W.W., and Gormley, W.B. 1999. Magnetoencephalographic mapping of the language specific cortex. J. Neurosurg. 90: 85-93.

Sarvas, J. 1987. Basic mathematical and electromagnetic concepts of the biomagnetic problem. Phys. Med. Biol. 32: 11-22.

Scoville, W.B. and Milner, B. 1957. Loss of recent memory after bilateral hippocampal lesions. J. Neurol. Neurosurg. Psychiatry 20: 11-21.

Shallice, T., Fletcher, P., Frith, C.D., Grasby, P., Frackowiak, R.S., and Dolan, R.J. 1994. Brain regions associated with acquisition and retrieval of verbal episodic memory. Nature 368: 633-635.

Simos, P.G., Breier, J.I., Zouridakis, G., and Papanicolaou, A.C. 1998. Assessment of functional cerebral laterality for language using magnetoencephalography. J. Clin. Neurophysiol. 15: 364-372.

Simos, P.G., Papanicolaou, A.C., Breier, J.I., Wheless, J.W., Constantinou, J.E., Gormley, W.B., and Maggio, W.W. 1999. Localization of language-specific cortex using MEG and intraoperative stimulation mapping. J. Neurosurg. 91: 787-796.

Stark, C.E.L. and Squire, L.R. 2000a. Functional magnetic resonance imaging (fMRI) activity in the hippocampal region during recognition memory. J. Neurosci. 20: 7776-7781.

- 2000b. fMRI activity in the medial temporal lobe during recognition memory as a function of study-test interval. Hippocampus 10: 329-337.

Wagner, A.D., Gabrieli, J.D.E., and Verfallie, M. 1997. Building memories: Remembering and forgetting of verbal experiences as predicted by brain activity. Science 281: 1188-1191.

Wright, A.A., Cook, R.G., Rivera, J.J., Shyan, M.R., Neiworth, J.J., and Jitsumori, M. 1990. Naming, rehearsal, and interstimulus interval effects in memory processing. J. Exper. Psychol.: L.M.C. 16: $1043-1059$.

Received September 26, 2001; accepted in revised form April 11, 2002. 


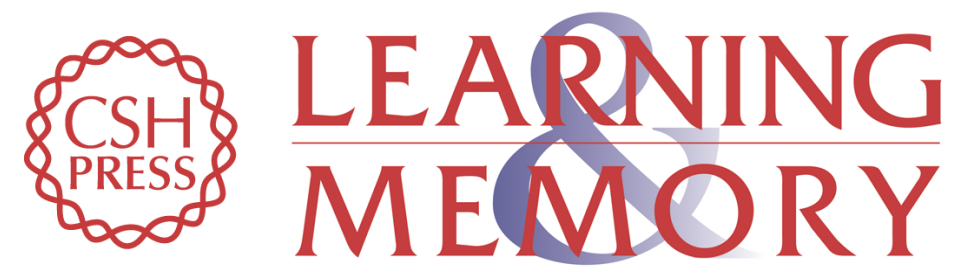

\section{The Hippocampus and Memory of Verbal and Pictorial Material}

Andrew C. Papanicolaou, Panagiotis G. Simos, Eduardo M. Castillo, et al.

Learn. Mem. 2002, 9:

Access the most recent version at doi:10.1101/lm.44302

References This article cites 26 articles, 8 of which can be accessed free at: http://learnmem.cshlp.org/content/9/3/99.full.html\#ref-list-1

License

Email Alerting Receive free email alerts when new articles cite this article - sign up in the box at the Service top right corner of the article or click here. 